\title{
Hybrid Algorithm for Image Retrieval using LBG and K-means
}

\author{
Seema Anand Chaurasia \\ Assistant Professor \\ Xavier Institute of Engineering \\ Mahim, Mumbai.
}

\author{
Vaishali Suryawanshi \\ Assistant Professor \\ Thadomal Shahni Engineering College, Bandra, \\ Mumbai, India.
}

\begin{abstract}
In this paper a hybrid algorithm for image retrieval based on texture feature extraction is proposed. Proposed algorithm can be implemented for texture feature retrieval using Vector Quantization (VQ). For texture feature retrieval Linde-BuzoGray (LBG) algorithms is used by dividing each image into pixel blocks of size $2 \mathrm{X} 2$ where each pixel consists of green, red and blue component. A training vector of dimension 12 can be obtained by putting these in a row. A training set is collection of such training vectors. Size of codebook will be 16X12.In the proposed method K-means algorithm is applied on existing LBG codebook and results are compared with LBG algorithm. From experiments it is found that proposed algorithm gives better relevance percentage as compared to the LBG algorithm.
\end{abstract}

\section{Keywords}

LBG Algorithm, K-Means Algorithm, Clustering, CBIR

\section{INTRODUCTION}

The steady growth of the Internet, the falling prices and easy availability of storage devices, and an increasing pool of available computing power make it necessary and possible to manipulate very large repository of digital information efficiently. Using content based Image retrieval one can search and browse in a large collection of digital image database based on automatically derived image features.

World Wide Web plays an important role in communication, education, industry etc. Size of digital image data is growing rapidly and hence it becomes very important to retrieve and store data efficiently [2, 4]. There are two main approaches for Image retrieval, one based on text based approach and second on content based Image retrieval.

Text based descriptor method is also known as image textual metadata. But there are two main disadvantages related to text-based approach. One is considerable amount of human labor is needed for manual annotation and second one is annotation inaccuracy due to human perception. This method lacks efficiency and simply not practical in databases where number of new images are growing rapidly. The need to locate these images and manage target images in response to user queries has become a very serious problem. Second

approach is Content based Image retrieval (CBIR)[5,6].CBIR is the process of retrieving desired images from a large collection of image database on the basis of color, texture and shape, which can be automatically derived from the images themselves[11,13]. Thus using a Content Based Image
Retrieval (CBIR), we can analyze and index images based on their visual contents [12].

One of the main applications of CBIR is category search. In category search user may have a group of images and search is for other additional images of same class or category. In other words, it may be the class that the user has examples and the search is for other elements of the same class. Categories may be derived from the labels or emerge from the database $[7,8]$.

\section{LITERATURE REVIEW}

Search time can be reduced considerably by using clustering $[9,10]$. LBG and K-means algorithms are some of the clustering methods which are most widely used. In this section LBG and K-means algorithms are explained.

\subsection{LBG Algorithm}

Consider two-dimensional vector space as shown in figure 1.In this algorithm centroid is computed as the first codevector $\mathrm{C} 1$ for the training set. Two codevector v1 and v2 are generated as shown in figure 1 by adding constant error to the codevector [1].

Two clusters are formed by Euclidean distance of all training vectors with vectors $\mathrm{v} 1$ and $\mathrm{v} 2$ based on nearest of $\mathrm{v} 1$ and $\mathrm{v} 2$. Four clusters are generated by repeating the same procedure for these two clusters and similarly eight clusters are generated by repeating the same procedure for these four clusters. This procedure is repeated for every new cluster until the required size of codebook is reached.

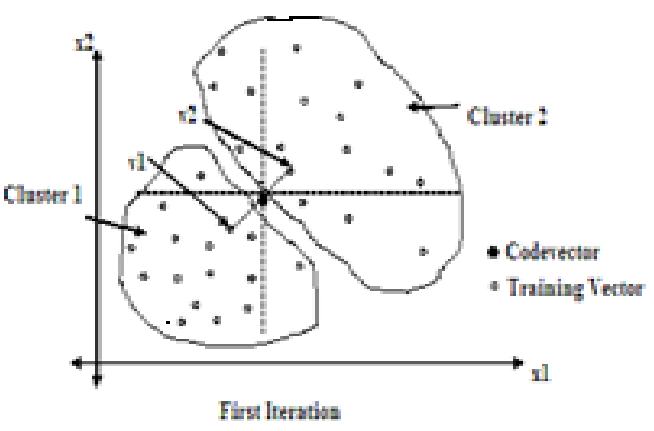

Fig 1: LBG algorithm for 2-dimensionl case 


\subsection{K-means on LBG codebook}

$\mathrm{K}$-means algorithm is applied on existing LBG codebook to create global codebook as follows [3]:

1. Obtain codebook containing $\mathrm{k}$ codevectors using LBG codebook generation algorithm.

2. Give the above LBG codebook as an input to Kmeans algorithm by considering first codebook of each category as seed codebook.

3. Find the squared Euclidean distance of all the training vectors with the $\mathrm{k}$ codevectors and $\mathrm{k}$ clusters are formed. A training vectors $\mathrm{Xj}$ is put in ith cluster if the squared Euclidean distance of the $\mathrm{Xj}$ with ith codevector is minimum. In case the squared Euclidean distance of $\mathrm{Xj}$ with codevectors happens to be minimum for more than one codevector then $\mathrm{Xj}$ is put in any one of them.

4. Compute centroid for each cluster.

5. Replace initial codevectors by the centroids of each cluster respectively.

6. Repeat the steps 3 to 5 for the respective number of LBG codebooks.

\section{PROPOSED ALGORITHM}

In this section proposed algorithm is explained. In this algorithm common codebook is created for both query images for each category and common codebook for each category containing all images of that category.

Proposed algorithm can be applied as follows:

1. Create global codebook of query images from each category by applying K-means algorithm on existing LBG codebook of query images.

2. Create global codebook for each category by applying K-means algorithm on existing LBG codebook.

3. Find Euclidean distance (similarity measure) ED1, ED2, ED3...ED N between global codebook of query images of each category and global codebook of all categories.

4. Sort Euclidean distance array (ED1, ED2, ED3 ....ED $\mathrm{N}$ ) in ascending order and save corresponding category number. Here $\mathrm{N}$ is total number of categories in image database.

5. Checking whether category number of global codebook of query images matches with the category with the smallest Euclidean distance and retrieve all the images of that category.

\section{SCHEME OF IIMPLEMENTATION}

For efficient image indexing and retrieval we are using the LBG algorithm as follows [1]:

1. To obtain LBG codebook image is first divided into the non-overlapping windows of size $2 \times 2$ pixels. (Each pixel consisting of red, green and blue components).

2. These are put in a row to get 12 coordinates per vector. A training set is collection of these vectors. (initial cluster).

3. Compute centroid (codevector) of the cluster.

4. Split the cluster using LBG.

5. Repeat the 3,4 till we obtain codebook of required size.
6. The codebook is stored as the feature vector for the image. Squared Euclidian distance is used as similarity measure.

To check the performance of proposed technique two measures are used i.e. precision and Recall [1].

$$
\begin{aligned}
& \text { Pr ecision }=\frac{\text { Number of relevant images retrieved }}{\text { Total number of relevant images }} \\
& \operatorname{Re} \text { call }=\frac{\text { Number of relevant images retrieved }}{\text { Total number of images in database }}
\end{aligned}
$$

After obtaining codebook using LBG algorithm, K-means algorithm is applied on existing LBG codebook to create global codebook of query images of each category and a single global codebook for all categories. Euclidean distance is calculated between each global codebook of query images and global codebook of all categories. Similarity measure will be based on squared Euclidean distance.

Fig.2. Shows a sample database of 100 images by randomly selecting one image from each category. The database has 100 categories, and each category consists of 72 images for a total of 7200 images. The image database used in the experiments is the subset of Columbia Object Image Library (COIL-100).

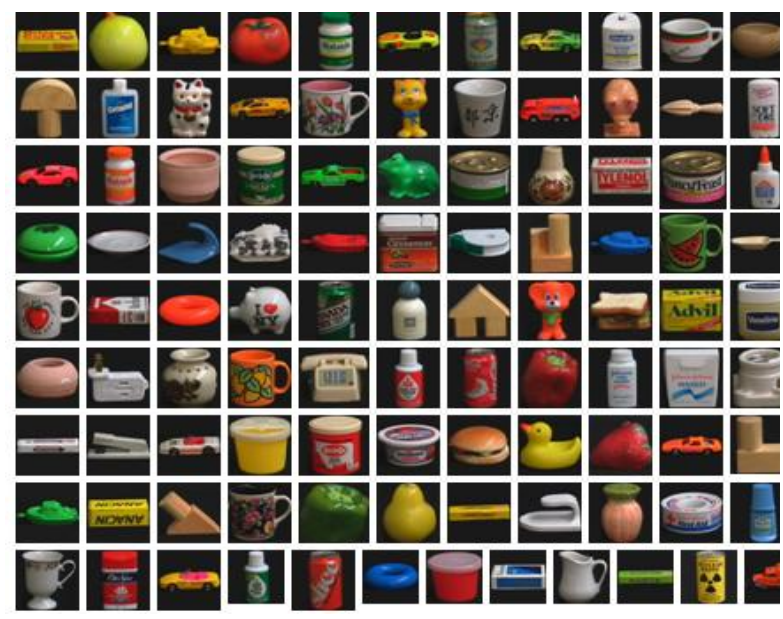

Fig 2: Sample database consisting of 7200 Images, the database has 100categories, 72 images in each category.

The method is implemented in Matlab 7.0 on Intel Core 2 Duo Processor T8100, $2.1 \mathrm{GHz}, 2 \mathrm{~GB}$ RAM machine to obtain results.

\section{RESULTS}

In this section results obtained using LBG algorithm and proposed algorithm are discussed.

\subsection{LBG Algorithm}

Experiments are carried out by varying number of categories each time and recording crossover point of precision and recall.

Fig 3 shows Precision and Recall graph based on LBG algorithm on 40 categories. 


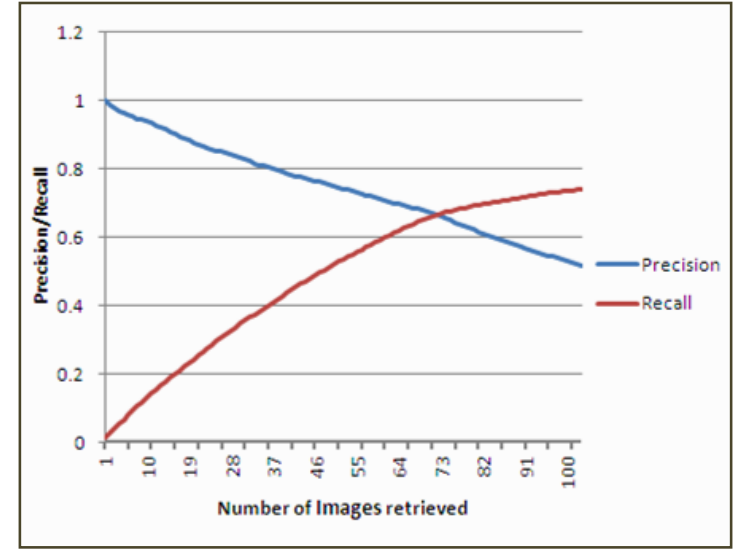

Fig 3: Plot of Precision/Recall Vs Total number of Images retrieved using LBG on color images on 40 categories Crossover point obtained is $66.66 \%$

Table 1 shows Crossover point of Precision and recall for different number of categories. It is seen from the table that crossover point decreases with the increase in number of categories.

Table 1: Crossover point of Precision/Recall for different number of categories using LBG algorithm

\begin{tabular}{|c|c|}
\hline Number of Categories & $\begin{array}{c}\text { Crossover Point of } \\
\text { Precision \& Recall (\%) }\end{array}$ \\
\hline 20 & $80.30 \%$ \\
\hline 30 & $67.90 \%$ \\
\hline 40 & $66.50 \%$ \\
\hline
\end{tabular}

\subsection{Proposed Algorithm}

Experiments are carried out by increasing number of categories and number of query images used in creating global codebook of query images. Table 2 shows Relevance percentage for different number of categories and query images.

Table 2: Relevance percentage for different number of categories using proposed algorithm

\begin{tabular}{|c|c|c|c|}
\hline \multirow{2}{*}{$\begin{array}{c}\text { No. of } \\
\text { categories }\end{array}$} & \multicolumn{3}{|c|}{ No. of Query Images } \\
\cline { 2 - 4 } & $\mathbf{5}$ & $\mathbf{1 0}$ & $\mathbf{1 5}$ \\
\hline 20 & $50 \%$ & $75 \%$ & $80 \%$ \\
\hline 30 & $43.33 \%$ & $53.33 \%$ & $66.67 \%$ \\
\hline 40 & $42.50 \%$ & $57.50 \%$ & $70 \%$ \\
\hline
\end{tabular}

\section{CONCLUSION}

Here in proposed method K-means algorithm is applied on existing LBG codebook of images. The efficiency of LBG codebook can be increased by using $\mathrm{K}$-means clustering algorithm. A global codebook of query images is created for each category by randomly selecting 5,10 and 15 query images from each category. A global codebook is created for each category containing all the images of that category by applying K-means algorithm on existing LBG codebook.
Matching is performed between each global codebook of query images and global codebook of all categories. The proposed system uses Euclidean distance as the similarity measure.

The proposed algorithm effectively reduces overall time complexity. The proposed algorithm effectively minimizes the undesirable results and gives a good relevance percentage by giving minimum number of non relevant images.

The LBG algorithm and proposed algorithm are performed by varying the database to different sizes. The performance evaluation of LBG algorithm is done by precision and recall and it is observed that, best retrieval results are achieved when size of database is less as compared to large database. The performance evaluation of proposed algorithm is done by observing number of relevant categories and it is observed that best results are achieved when global codebook of query images from each category is made from more number of images.

\section{REFERENCES}

[1] Dr. H.B. Kekre, Dr. Tanuja K. Sarode, Sudeep D. Thepade, Vaishali Suryavanshi, "Image retrieval using Texture features extracted from GLCM, LBG and KPE". International Journal of computer Theory and Engineering, Vol.2, No. 5, October 2010

[2] Dr. H.B.Kekre ,Dr. Tanuja K. Sarode,'New Clustering Algorithm for Vector quantization using Rotation of error vector". international Journal Of computer Science and Information Security, Vol.7,no 3, 2010.

[3] Dr. H.B .Kekre, Dr. Tanula K.Sarode,"Vector Quantized Codebook Optimization using K-means" International Journal on Computer Science and Engineering Vol.1(3), 2009, 283-290

[4] Bang Huang, Linbo Xie "An Improved LBG algorithm for Image Vector 8-1-4244-5540-9,2010 IEEE.

[5] Tejas P. Kokate,"Cluster-based Image Retrieval Techniques" .IJECS Volume 2 Issue 5 May, 2013 Page No. 1474-1478.

[6] Akash Saxena, Sandeep, Saxena, Akanksha Saxena, "Image Retrieval using Clustering Based Algorithm". International Journal Of Engineering Vol. 1, Issue 3 September 2012

[7] Hossein Nezamabadi-pour and Saeid Saryazdi, "Indexing and Retrieval in DCT Domain using Clustering Techniques". World Academy of Science, Engineering and Technology 32007.

[8] Arnold W.M. Smeulders, Marcel Worring, Simone Santini, "Content-based image retrieval at the end of the early years".IEEE Transactions on Pattern analysis and machine intelligence, vol 22, No 12, December 2000.

[9] Harikrishna Narasimhan, Purushothaman Ramraj,"Contribution-Based Clustering Algorithm for Content-Based Image Retrieval”, Ijarcsse, Vol 2, 2011.

[10] A.Kannan,Dr.V.Mohan,Dr.N.Anbazhagan” Image Clustering and Retrieval using Image Mining Techniques"International Conference on Computational Intelligence and Computing Research 2010 IEEE. 
[11] H.B.Kekre, Sudeep D. Thepade, "Rendering Futuristic Image Retrieval System", National Conference on Enhancements in Computer,Communication and Information Technology, EC2IT-2009, 20-21 March 2009, K.J.Somaiya College of Engineering, Vidyavihar,Mumbai-77.

[12] Dr. H.B.Kekre ,Dr. Tanuja K. Sarode,’New Clustering Algorithm for Vector quantization using Rotation of error vector". international Journal Of computer Science and Information Security, Vol.7,no 3, 2010.

[13] H.B.Kekre, Sudeep D. Thepade, "Image Retrieval using Augmented Block Truncation Coding Techniques", ACM Int. Conference on Advances in Computing, Comm. and Control (ICAC3-2009), pp.384-390, 23-24 Jan 2009, Fr. CRCE, Mumbai. Is uploaded at ACM portal. 